\title{
A Computational Approach to Design High Reliability Ceramic Laminates
}

\author{
E. Bertarelli, D. Gastaldi, P. Vena* and R. Contro
}

Politecnico di Milano, Department of Structural Engineering, Laboratory of Biological Structure Mechanics Piazza Leonardo da Vinci, 32, 20133 Milano - Italy

\begin{abstract}
A numerical design approach for ceramic multilayer laminates with predetermined stress profiles is here proposed and compared with a well established theoretical method. The design procedure allows for the optimization of layer composition, in order to obtain high reliability and at the same time control composition and stress state of each layer.

The proposed approach is used to design alumina-mullite-zirconia laminates with high toughness, meeting at the same time the particular functional requirements of a pure alumina surface layer with residual compressive stresses with the aim to increase tribological properties.
\end{abstract}

Keywords: Functionally graded materials, ceramic laminates, linear elastic fracture mechanics, finite element method, biomedical applications.

\section{INTRODUCTION}

The main limitation to the use of ceramic materials in structural applications is owed to their low reliability, i.e. their high scatter in failure strength and low fracture toughness. This behavior is directly related to the presence of flaws originated during the production processes and the service. Although improvements in material quality, manufacturing methods and component design have resulted in a drastic reduction of structure failures, the low fracture toughness of ceramics, coupled with their susceptibility to failure by slow crack growth under stresses well below their fracture strength, remains a concern for the reliability of ceramic bearings in many engineering applications [1].

Many efforts have been made with the purpose to increase the reliability of ceramic materials: a promising approach is the design and manufacturing of multilayered structures with a tailored residual stress field. Layers of ceramic composites with different composition are stacked, obtaining a graded ceramic, with the purpose to develop a specific residual stress profile as a result of the sintering process. The thermal stresses are due to the mismatch of the mean coefficient of thermal expansion between the layers and between the different grains in the same layer $[2,3]$.

It was shown that in ceramic multilayer laminates with alumina surface layer, a compressive residual stress at the surface has beneficial effects on the wear and friction behavior, as a consequence of the reduction of crack nucleation and propagation and the formation of thinner and smoother tribolayers [4].

Similarly, a compressive layer placed at a certain depth can significantly increase the toughness of the material [5].

\footnotetext{
*Address correspondence to this author at the Department of Structural Engineering, Laboratory of Biological Structure Mechanics, 32, 20133 Milano - Italy; Tel: +39.02.2399.4236; Fax: +39.02.2399.4220;

E-mail: vena@stru.polimi.it
}

Indeed, the compressive stress required to achieve high strength is often very high and localized and delamination between layers (i.e. edge cracking) may occur [6].

Recently, in [7] has been proposed the design and manufacturing of Alumina/Mullite/Zirconia multilayer laminates with an engineered residual stress profile with a maximum compression at a certain depth from surface, obtaining a lowscattered strength and a higher reliability in comparison to monolithic ceramics. The Authors applied numerical methods to study the fracture process of ceramic laminates through both energy considerations and statistical approaches $[8,9]$.

In the present work the Authors present a numerical approach to the design of ceramic laminates exhibiting high reliability and improved strength. The main idea is to use computation models for the assessment of mechanical response of laminates with predefined mechanical properties, with the purpose to obtain specific stress profiles which result in improved component performances.

In this paper this strategy is applied, following prescriptions from [4] for ceramic FGM in bio-implants (artificial joints). Thus, we propose optimized laminate structure, with

- pure alumina surface layer with compressive residual stresses, for a better tribological behavior;

- an effective residual stress profile, with the aim to obtain an high fracture toughness;

- low zirconia content in internal layers, to limit the residual stresses leading to crack formation.

\section{EFFECTIVE TOUGHNESS AND STRENGTH OF CERAMIC LAMINATES}

In this paper, nine layer symmetric multilayer laminates with different layer composition have been considered, with the shape of bars having nominal length of $60 \mathrm{~mm}$ and a rectangular cross section $(7.5 \mathrm{~mm}$ height and $1.45 \mathrm{~mm}$ thickness). 
Table 1. Material Properties

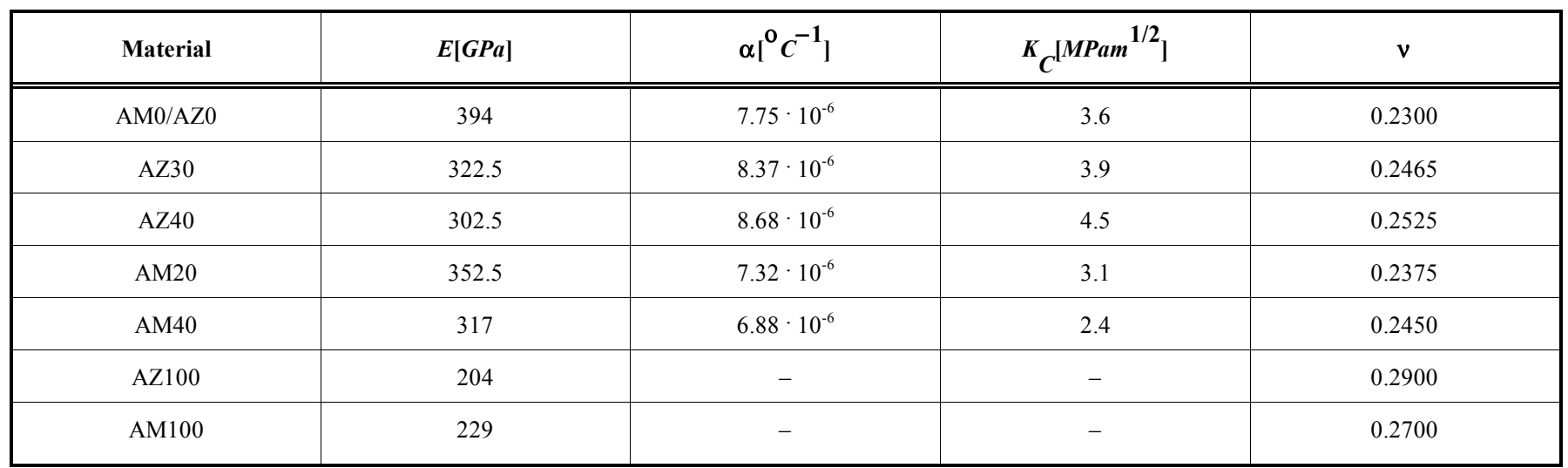

Composite materials with Alumina, Mullite and Zirconia constituents were considered. Thickness and material compositions of layers are reported in Fig. (1) for the reference laminate $A M Z-1$ (from [7]) and proposed alternate structures $A M Z-2$ and $A M Z-3$. Following notation reported in [7] each layer is identified as $A Z w / y$ or $A M w / y$ in which $A, Z$ and $M$ stand for Alumina, Zirconia and Mullite, respectively; $w$ is the volumetric content of Zirconia or Mullite and $y$ is the layer thickness in $\mu \mathrm{m}$.

The elastic constitutive parameters, i.e. the Young modulus and the Poisson ratio, as well as the coefficient of thermal expansions of the composites at different volumetric compositions used in [10] have been assumed (see Table 1). The Young modulus and Poisson ratio were estimated through the Voigt and Reuss bounding values. The thermal expansion coefficient and fracture toughness for $A M$ and $A Z$ composites were measured on monolithic samples [11].

First of all, the theoretical approach proposed by [7] to evaluate the effective toughness is reported. Subsequently, the presented finite element model is compared with theoretical one referring to $A M Z-1$ structure, giving insight on its capabilities to study in details mechanical behavior of laminates.

In conclusion, this numerical method is used to design most effective laminate structures with different layer compositions (i.e. $A M Z-2$ and $A M Z-3$ structures).

\section{THEORETICAL APPROACH}

In the Linear Elastic Fracture Mechanics (LEFM) framework, a stable crack propagation, at a given crack length $(c)$, happens if i) the stress intensity factor $K_{\text {ext }}(c)$ resulting from external applied loads equals the fracture toughness $T$; and ii) the function $T(c)$ is steeper than $K_{\text {ext }}(c)$.

Analytically, this is expressed by:

$K_{\text {ext }}=T(c)$

$\frac{d K_{e x t}}{d c}<\frac{d T(c)}{d c}$

It's worth to underline that the LEFM model holds when the process zone, in which fracture surfaces mechanically interacts, and the area of permanent deformations ahead of the crack tip are accounted to be small. This hypothesis is generally accepted for ceramic materials if grain interlocking and phase transformation processes are negligible with respect to other phenomena like those induced by residual stresses. In this respect, all involved phenomena, here studied, are linear and the superposition principle applies.

Accounting residual stresses, in a multilayer laminate the propagation of a crack occurs when

$K_{\text {ext }}+K_{\text {res }}=K_{C, i}$
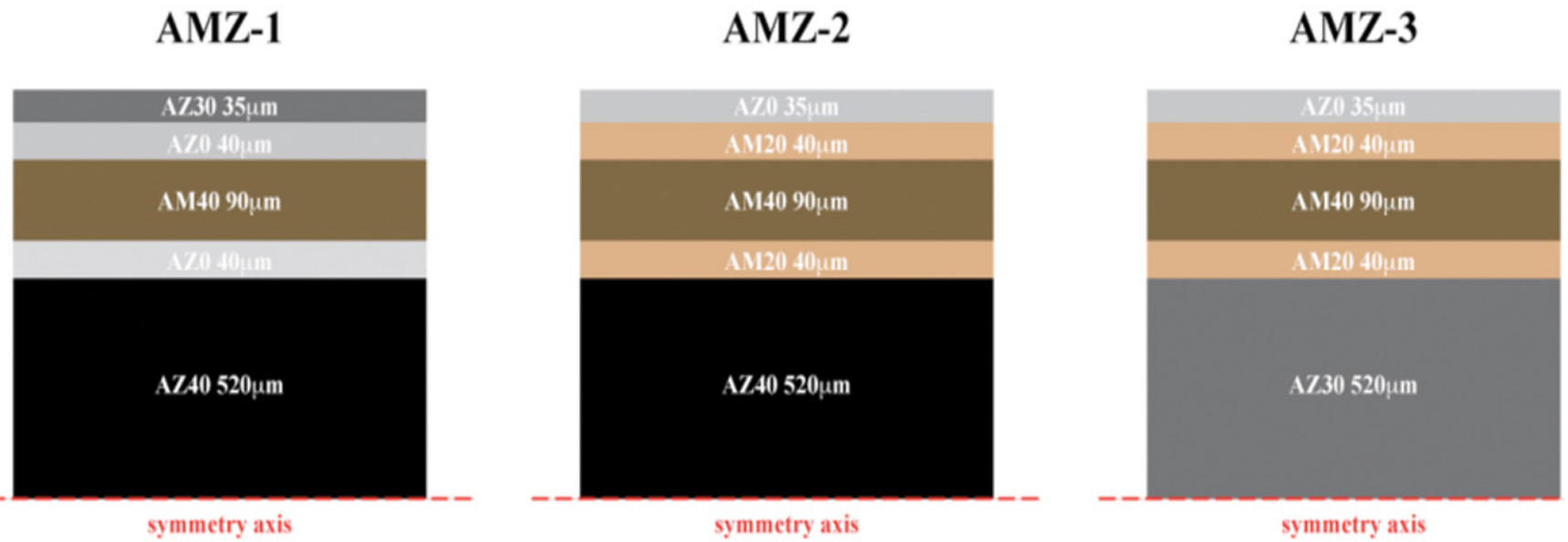

Fig. (1). Architecture of the $A M Z$ laminates (not to scale): reference $A M Z-1$ and proposed $A M Z-2$ and $A M Z-3$. 
i.e. when the sum of $K_{\text {ext }}$ and $K_{\text {res }}$, which represents the stress intensity factor due to residual stress profile, equals the intrinsic fracture toughness of the $i$-th layer $K_{C, i}$. This way, including the residual stresses as a part of the crack propagation resistance of the material, we can define the apparent fracture toughness as

$T^{i}=K_{C, i}-K_{\text {res }}$

Considering a crack propagating in a semi-infinite body along $x$ direction, starting from the surface and moving perpendicular to interfaces, accounting only mode I loading, we are able to write the simplified relation [7]

$K_{\text {res }}=\frac{Y(c)}{\sqrt{\pi c}} \int_{0}^{c} \sigma_{\text {res }}(x) \frac{2 c}{\sqrt{c^{2}-x^{2}}} d x$

with $Y \cong 1.1215$. Solving (5), for the superposition principle we have then in a generic multilayer laminate

$T^{i}=K_{C, i}-\sum_{j=1}^{i}\left[2 Y \sqrt{\frac{c}{\pi}} \Delta \sigma_{R, j}\left(\frac{\pi}{2}-\arcsin \left(\frac{x_{j}-1}{c}\right)\right)\right] \quad x_{i-1}<x<x_{i}{ }^{(6)}$

where the sum is performed over every layer interested by crack propagation until the $i$-th, where the crack tip is located and $\Delta \sigma_{R}$ indicates amplitude of residual stress step between layers [12].

It's worth to underline that in equation (6) the elastic modulus is assumed to be the same in each layer, accounting an error on estimating fracture toughness less than $10 \%$ if the Young modulus variation amongst layer is less than 33\% [6].

\section{NUMERICAL APPROACH}

The numerical method we propose to evaluate fracture behavior of laminate adopt a plane strain model to simulate the cooling process after sintering and therefore a standard four point bending tests [9]. Thus, the residual stress field is obtained by solving the finite element equations in which a thermal expansion, proportional to a temperature variation from stress-free temperature to room temperature $\Delta \mathrm{T}=-(1200-25){ }^{\circ} \mathrm{C}$, is applied. The coefficients of thermal expansion of the materials are reported in Table $\mathbf{1}$.

The effective toughness of the symmetric laminate subjected to residual stress was obtained through a series of finite element analyses at increasing crack length from a minimum of $0 \mu \mathrm{m}$ up to a maximum length of $250 \mu \mathrm{m}$.

A two step procedure is used to determine the effective toughness [5]: i) a series of analysis with increasing crack length in which external loads simulating the four point bending test with no residual stress is simulated and ii) a series of analysis with increasing crack length in which thermal expansion without external load is simulated. The crack propagation along the symmetry plane was simulated by progressively releasing the nodal constraints.

In the first series of analysis a unit force is used and a stress intensity factor due to external force is found by calculating the energy release rate $G(c)$ (load controlled test):

$G=-\frac{\partial \Pi}{\partial c}=\frac{\partial U}{\partial c}$ in which $\Pi$ is the total potential energy, $U$ is the elastic energy of the system and $c$ the current crack length. Assuming a mode $I$ crack propagation, the stress intensity factor $K^{\sigma}$ for a unit force is:

$K^{\sigma}=\sqrt{G(c) \frac{E}{1-v^{2}}}$

The above expounded finite element procedure has been validated through a quantitative comparison with results obtained through contour integrals presented on a different ceramic laminate in $[8,13]$.

In the second series of analysis, the stress intensity factor in the laminate subjected to residual stress $K_{\text {res }}(c)$ is calculated through the same procedure based on nodal constraint release as above expounded.

Due to linearity of the constitutive laws and to the linearity of the strain-displacements relationships, the principle of superposition of the solution from the thermal loading and that from the force loading can be used to determine the total stress intensity factory applied to the laminate subjected to the residual stress field and to the four point bending external loads $K(c)$ :

$K(c)=K_{\text {res }}(c)+K^{\sigma}(c) F$

in which $F$ is the force applied in the four point bending loading condition (Fig. 2).

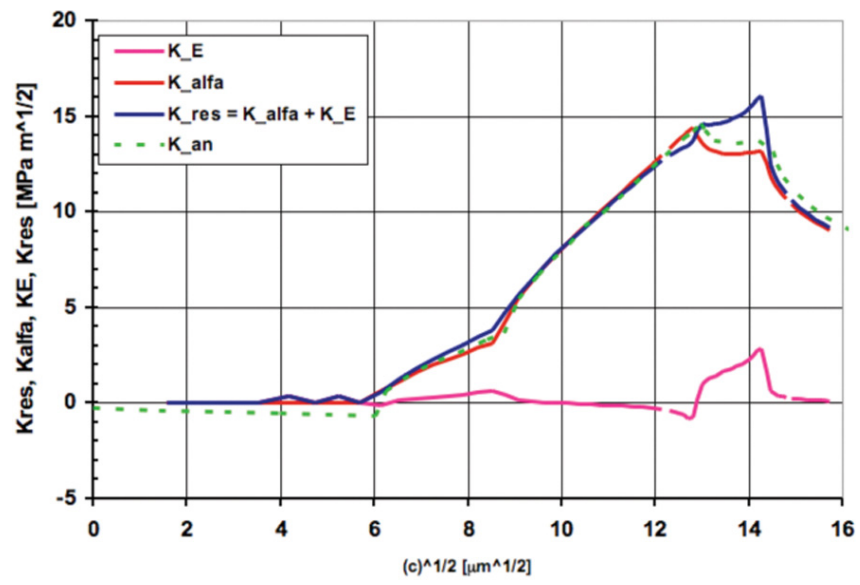

Fig. (2). Apparent fracture toughness of reference laminate $A M Z-1$.

The crack in the layer $n$ propagates when the condition

$K(c)=K_{C, n}$

is met, in which $K_{C, n}$ is the intrinsic fracture toughness of the composite material in the layer $n$. Combining the relationships (9) and (10) the effective toughness $K_{\text {eff }}(c)$ is obtained:

$K_{\text {eff }}=K^{\sigma}(c) F_{c r}=K_{C, n}-K_{r e s}(c)$

in which $F_{c r}$ is the force at which crack propagate.

\section{RESULTS AND DISCUSSION}

\subsection{Reference Laminate}

In Fig. (2) the apparent fracture toughness for the $A M Z-1$ laminate is reported. Dashed line represents analytical solu- 
tion, while the solid black line is obtained through the finite element simulation.

Different curves are obtained, because in the analytical solution constant elastic parameters $(E, v)$ are assumed (see equation [6]), while in the numerical model all thermomechanical parameters are set for each layer $\left(\alpha_{i}, E_{i}, v_{i}\right)$. A numerical simulation performed defining constant Young's modulus and Poisson's ratio (obtained as weighted average) confirmed this statement, leading a toughness curve very similar to the analytical one (solid red line in Fig. 2).

We found then that a variation of the Poisson's ratio has almost no effect [15].

Once again, in the linear elastic fracture mechanics framework, thanks to linearity of the constitutive laws and the strain-displacements relationships, the principle of superposition can be used for $K$. Thus, it's possible to split the stress intensity factor in the laminate subjected to residual stress $K_{\text {res }}$ in two distinct contributes, rewriting relation [4] as

$T^{i}=K_{C, i}-\left(K_{\alpha}+K_{E}\right)$

where $K_{\alpha}$ is the effect on laminate toughness of variation of thermal expansion coefficient among different layers, while $K_{E}$ is the effect of variation of elastic modulus only. In Fig. (2), the solid pink line represents the contribution $K_{\alpha}$.

According to [13], the variations of residual stresses produce much greater effect on toughness than the variation of elastic modulus. Nevertheless the $K_{E}$ contribute should not be neglected, and it should be distinguished from $K_{\alpha}$.

We found that for cracks approaching a stiffer material (i.e., with highest Young's modulus) the component $K_{E}$ is positive, leading to a crack shielding effect, thus a beneficial effect. For cracks growing towards more compliant materials (i.e., lowest Young's modulus) the $K_{E}$ component generates an increase of stress intensity factor (according to [14] and [15]).

Normalizing toughness with respect the shape factor $\Psi$, we obtain the toughness as material property (Fig. 3). The geometrical factor $\Psi$ can be calculated applying empirical expressions [13].

The effective stress intensity factor exhibited for $A M Z-1$ shows an increasing trend in the layers characterized by a compressive residual stress. After having reached a maximum value when the crack tip is located beyond the interface between the $A Z O$ and $A Z 40$ layers the curve decreases as a consequence of the tensile residual stress. The critical crack length found is approximately $169 \mu \mathrm{m}$.

Moreover, it's possible to identify two characteristic stress levels. For small magnitude stresses, the $K_{\text {ext }}(c)$ curve may intercept the $K_{\text {eff }}(c)$ curve for two values of $c$ say $c_{l}$ and $c_{2}$ : any crack shorter than $c_{1}$ does not propagate and cracks with $c_{1}<c<c_{2}$ propagates until they reach the length $c_{2}$.

Through this procedure, a critical value of the applied stress for which $c_{2}=c_{c r}$ can be identified: for tensile bending stresses $\sigma_{\max }^{t h, 1}>693 \mathrm{MPa}$ the crack propagates unstably.
The average strength obtained through the four point experimental tests is $692 \pm 25 \mathrm{MPa}$ [7], with a noticeable agreement. For tensile bending stress $\sigma_{\min }^{t h}<342 \mathrm{MPa}$, on the other hand, no propagation is expected except for very long cracks which have no practical interest.

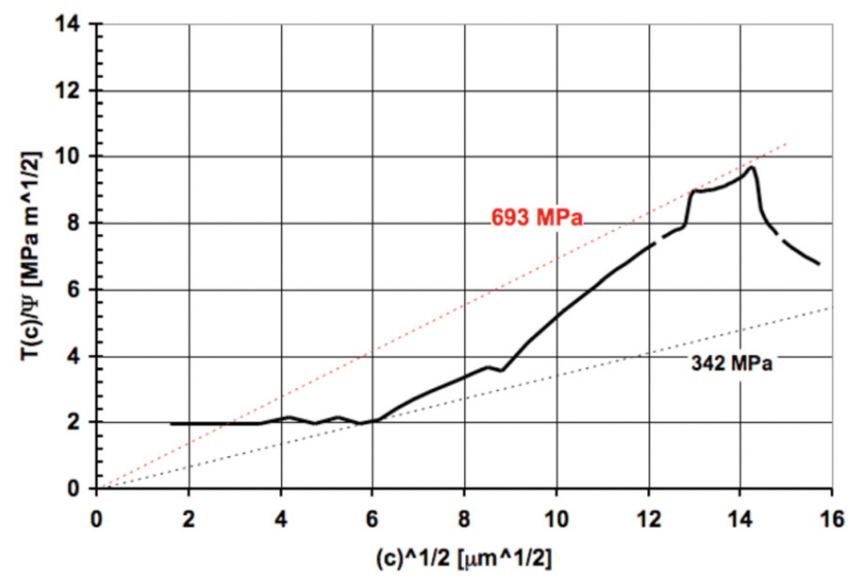

Fig. (3). Apparent fracture toughness of reference $A M Z-1$ structure. The dashed lines indicates $\sigma_{\max }^{\text {th }, 1}$ (red one) and $\sigma_{\min }^{\text {th, }}$ (black one).

\subsection{Design of New Structures Applying Numerical Ap- proach}

The subsequent step is the application of the above expounded design approach. We propose two alternate laminates $A M Z-2$ and $A M Z-3$, with the same structure and layer thickness, but with different layer composition (see Fig. 1). For these structures, the apparent fracture toughness has been calculated applying the numerical approach (Figs. 4,5).

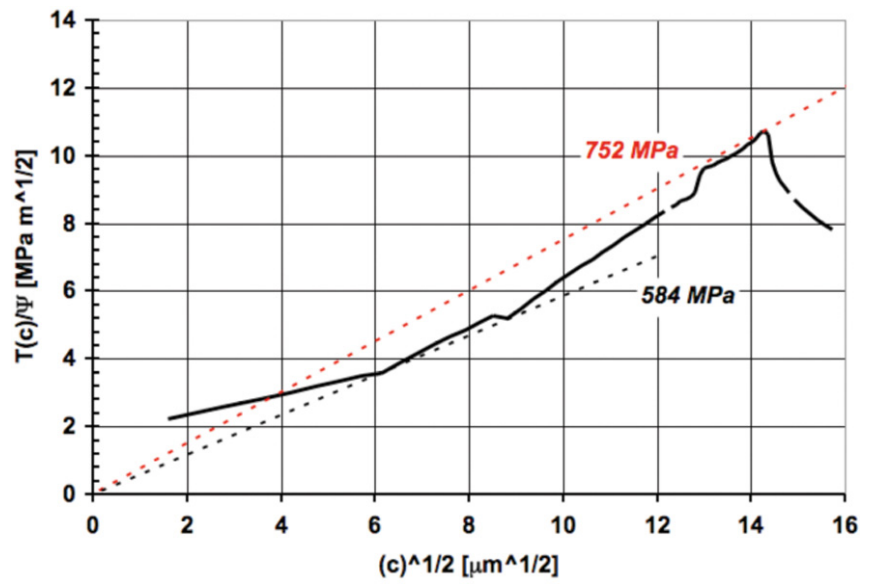

Fig. (4). Apparent fracture toughness of $A M Z-2$ structure. The dashed lines indicates $\sigma_{\max }^{t h, 2}$ (red one) and $\sigma_{\text {min }}^{t h, 2}$ (black one).

External layer in laminates $A M Z-2$ and $A M Z-3$ is pure alumina with compressive residual stresses, to obtain better tribological behavior. While in $A M Z-2$ the core contains $40 \%$ of zirconia, in $A M Z-3$ we reduced the amount of zirconia at $30 \%$, with the aim of limit the residual stresses that may lead to crack formation (see Fig. 6). 


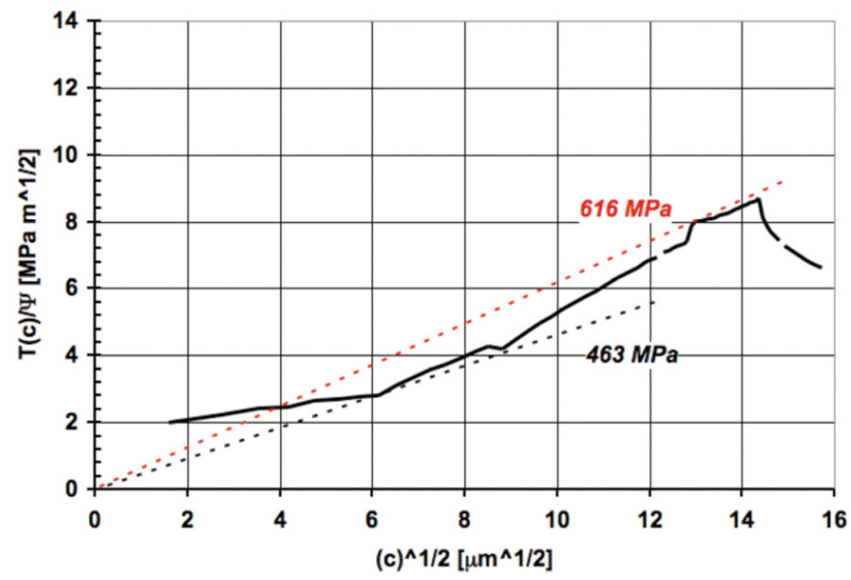

Fig. (5). Apparent fracture toughness of $A M Z-3$ structure. The dashed lines indicates $\sigma_{\max }^{\text {th }, 3}$ (red one) and $\sigma_{\text {min }}^{\text {th }, 3}$ (black one).

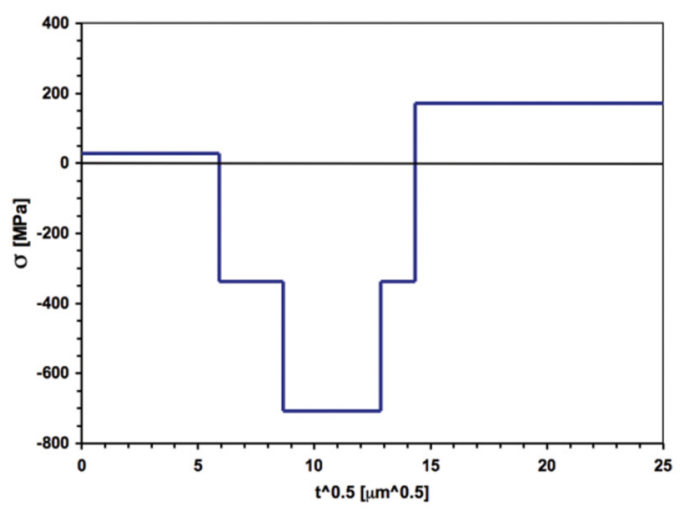

(a)

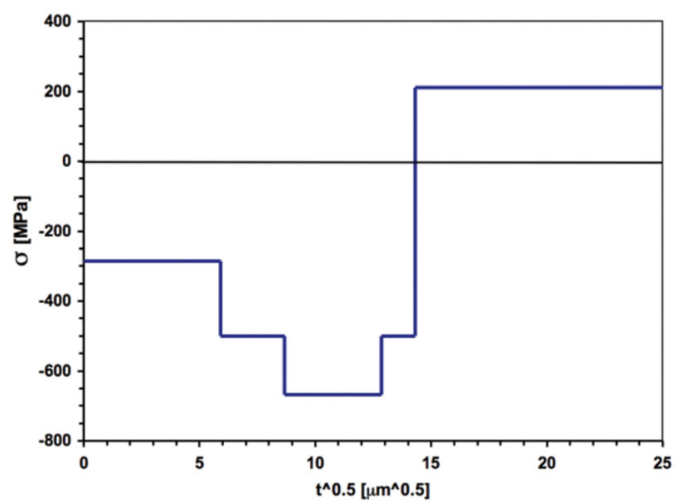

(b)

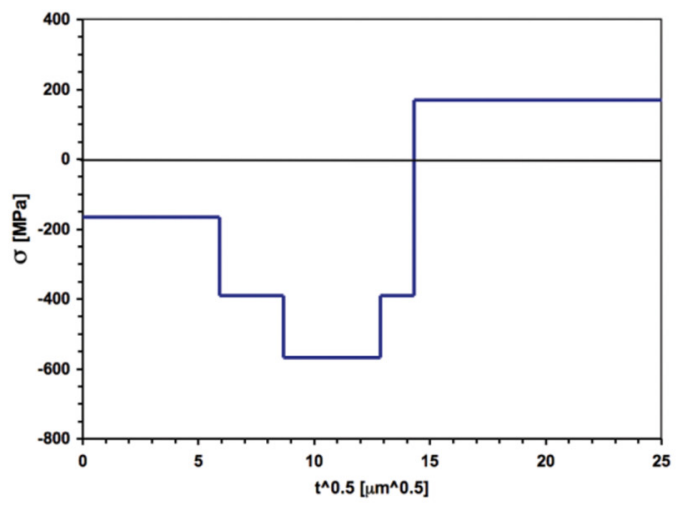

(c)

Fig. (6). Residual stress profiles for $A M Z-1$ (a), $A M Z-2$ (b) and $A M Z-3$ (c) structures.
Adopting the proposed numerical approach, we found for $A M Z-2 \sigma_{\max }^{t h, 2}=752 \mathrm{MPa}$ and $\sigma_{\text {min }}^{t h, 2}=584 \mathrm{MPa}$, and a compressive residual stress in the surface layer $\sigma_{\text {comp }}=-287$ $\mathrm{MPa}$. For $A M Z-3$, we found $\sigma_{\max }^{t h, 3}=616 \mathrm{MPa}$ and $\sigma_{\min }^{t h, 3}=$ $463 \mathrm{MPa}$, and a compressive residual stress in the surface layer $\sigma_{\text {comp }}=-165 \mathrm{MPa}$.

\section{CONCLUSIONS}

A numerical design approach for high reliability of ceramic laminates is here proposed. Numerical and theoretical solutions are compared, underlining advantages of the former one: it allows to explicitly account of the $K_{\alpha}$ and $K_{E}$ contribution, that represents the effect on laminate toughness of variation of thermal expansion coefficient, and the effect due to the variation of elastic modulus, respectively. The contribute $K_{E}$ is usually neglected in analytical solution, because of the necessary simplifying hypothesis of constant elastic modulus among layers.

Subsequently, the numerical design approach here proposed is used to optimize layers' composition, evaluating different laminate structures, in order to obtain an high reliability and at the same time to control composition and stress state of each layer with the purpose to match particular functional and structural requirements.

The high reliability of the laminate is achieved by designing the residual stress profiles with the purpose to grant high fracture toughness and two thresholds on the applied stress ( $\sigma_{\min }^{\text {th }}$ and $\sigma_{\max }^{\text {th }}$ ) which defines the range of the stable crack propagation. If the difference between these reference stresses is reduced, we should be able to find a most determined mechanical behavior of ceramic laminate.

The described approach can be used in future to improve the design of multilayer composites with arbitrary shape and loading conditions. Further experimental evaluations are needed to verify the effective behavior of proposed solutions, evaluating both mechanical and tribological aspects.

\section{REFERENCES}

[1] Rahaman MN, Yao A, Bal BS, Garino JP, Ries MD. Ceramics for Prosthetic Hip and Knee Joint Replacement. J Am Ceram Soc 2007; 90: 1965-88.

[2] Green DJ, Cai PZ, Messing GL. Residual stresses in AluminaZirconia laminates. J Eur Ceram Soc 1999; 19: 2511-7.

[3] Sergo V, Lipkin M, De Portu G, Clarke DR. Edge stresses in Alumina-Zirconia laminates. J Am Ceram Soc 1997; 80: 1633-8.

[4] Novak S, Kalin M, Lukas P, Anne G, Vleugels J, Van Der Biest O. The effect of residual stresses in functionally graded alumina-ZTA composites on their wear and friction behavior. J Eur Ceram Soc 2007; 27: 151-6.

[5] Vena P, Gastaldi D, Contro R. Effects of the thermal residual stress field on the crack propagation in graded Alumina-Zirconia ceramics. Mater Sci Forum 2005; 492-493: 177-82.

[6] Moon RJ, Hoffman M, Hilden J, Bowman KJ, Trumble KP, Rödel $\mathrm{J}$. R-curve behavior in Alumina-Zirconia composites with repeating graded layers. Eng Fract Mech 2002; 69: 1647-65.

[7] Sglavo VM, Bertoldi M. Design and production of ceramic laminates with high mechanical resistance and reliability. Acta Mater 2006; 54: 4929-37.

[8] Bertarelli E. Master's Thesis, Politecnico di Milano, in italian 2007.

[9] Vena P, Bertarelli E, Gastaldi D, Contro R. Energy-based and local approaches to the strength analysis of ceramic laminates with ther- 
mal residual stresses through the finite element method. Mech Res Comm, in press; 2008.

[10] Sglavo VM, Larentis L, Green JL. Flaw insensitive ion-exchanged glass: I, theoretical aspects. J Am Ceram Soc 2001; 84: 1827-31.

[11] Bertoldi M, Paternoster M, Sglavo VM. Production of multilayer ceramic laminates with improved mechanical properties. Ceram Trans 2003; 153: 89-102.

[12] Sglavo VM, Bertoldi M. In: Proceedings of the 29th international conference on advanced ceramics and composites, Cocoa Beach (FL), Am Ceram Soc; 2005.
[13] Chen CR, Pascual J, Fischer FD, Kolednik O, Danzer R. Prediction of the fracture toughness of a ceramic multilayer composite Modelling and experiments. Acta Mater 2007; 55: 409-21.

[14] Becker TL Jr, Cannon RM, Ritchie RO. Statistical fracture modelling: crack path and fracture criteria with application to homogeneous and functionally graded materials. Eng Fract Mech 2002; 69: $1521-55$.

[15] Simha NK, Fischer FD, Kolednik O, Chen CR. Inhomogeneity effects on the crack driving force in elastic and elastic-plastic materials. J Am Ceram Soc 2003; 51: 209-40.

(C) Bertarelli et al.; Licensee Bentham Open.

This is an open access article licensed under the terms of the Creative Commons Attribution Non-Commercial License (http://creativecommons.org/licenses/by-nc/3.0/) which permits unrestricted, non-commercial use, distribution and reproduction in any medium, provided the work is properly cited. 